\title{
Statistical particle stress in aeolian sand movement-derivation and validation
}

\author{
Yu Zhang *, Yaojian Li, Jiecheng Yang, Dayou Liu \\ Laboratory of Environmental Mechanics, Institute of Mechanics, Chinese Academy of Sciences, Beijing 100190, China
}

\section{A R T I C L E I N F O}

\section{Article history:}

Received 23 September 2010

Received in revised form 17 December 2010

Accepted 27 January 2011

Available online 18 February 2011

Keywords:

Particle group

Statistical particle stress

\begin{abstract}
A B S T R A C T
The statistical particle stress (SPS) is a result of group particle movement, which is not referred directly from E-L (Euler-Lagrange) calculations. This paper derives SPS in Eulerian regime and proves that in aeolian sand movement, as the height increases the gas stress also increases while the SPS decreases; however, the sum of gas stress and SPS keeps to be a constant value, which equals to the gas stress in particle absent region. This paper also suggests that SPS predominates in the momentum transportation of particle phase.
\end{abstract}

(c) 2011 Elsevier B.V. All rights reserved.

\section{Introduction}

Two-phase flow can be described by either the Eulerian-Eulerian (E-E) model or the Eulerian-Lagrangian model(E-L). In the E-E model, the particle phase is treated as a pseudo-fluid, and it also has stress, pressure as well as other characteristics that only continuum possesses. On the contrary, the E-L model treats the particle phase as a discrete phase, and tracks movement of individual particle that obeys Newton's second law of motion. It is noteworthy that the concept of statistical particle stress (SPS) is not involved in the E-L model.

The E-E model greatly saves computational time. However, additional models are needed to close the conservation equations of the particle phase. Unreasonable models may result in unreasonable predictions. When doing E-L simulation, a huge amount of computational data is obtained, including information of every particle's trajectory $\mathbf{r}_{s}(t)$ (or $x_{s}(t), y_{s}(t), z_{s}(t)$ ) and velocity $\mathbf{v}_{s}(t)$ (or $u_{s}(t), v_{s}(t), w_{s}$ $(t))(s=1,2,3, \cdots \cdots)$. Those results give details of an individual particle's movement rather than the statistical regularity. However, the later one is always more concerned by researchers. For example, in aeolian sand movement research, a high-speed photography method was used to study particle saltation movement in the wind tunnel, and the results showed that the distribution of particle velocity along the height followed the power function pattern [1]. The sand flux above the sand bed surface was measured with a sand trapper, and the results showed that the exponential function can describe the variation of sand flux with height [2]. The probability distributions of particle impact and lift-off velocities on bed surface and the particle velocity distributions at different heights in a wind tunnel were measured in details with PDPA (Phase Doppler Particle Analyzer) measurement technology [3]. Consequently, in order to

\footnotetext{
* Corresponding author. Tel.: +86 10 82544231; fax: + 861062561284 . E-mail address: zhangyu@imech.ac.cn (Y. Zhang).
}

compare CFD (computational fluid dynamics) simulation results with experimental data, the statistical post-process should be performed after getting E-L simulation results, from which one can be obtained that averaged particle concentration $\alpha_{\mathrm{p}, i j k l}=\left(\frac{\pi}{6} d_{\mathrm{p}}^{3} N_{i, j, k, l}\right) / V_{i, j, k}$, averaged particle velocity $\overline{\mathbf{v}}_{\mathrm{p}, j \mathrm{jkl}}=\left(\sum_{\mathbf{r}_{\mathbf{s}} \in \mathbf{V}_{\mathbf{i}, \mathbf{k}, \mathbf{k}}} \mathbf{v}_{\mathbf{s}}\left(\mathbf{t}_{\mathbf{l}}\right)\right) / N_{i, j, k, l}$ and other averaged properties, where $N_{i, j, k, l}$ is the number of particles that locate inside a grid $V_{i, j, k}$ at a moment $t_{l}$. The aeolian sand sediment process was numerically studied, and the simulation results were statistically summarized and compared with experimental results $[4,5]$. The particle collision process occurring at dense particle zone was simulated by the E-L method, and the probability function of particle movement after collision was obtained [6]. However, those averaged results from E-L simulations are still not good enough to explain the transportation process in particle phase. It only shows the similar statistical regularity from experimental results. CFD work should be able to do more theoretical analysis. Therefore, a new concept-SPS is introduced in this paper to derivate its mathematical formulation, and to discuss its magnitude in analysis of aeolian sand movement. The further objective of this research is to develop a reasonable E-E model, which solves global particle movement by integration SPS in particle momentum transportation equation.

\section{Derivation of statistic particle stress}

Fig.1 gives the calculation domain of aeolian sand movement. When the flow reaches steady and fully developed state, $\partial / \partial t=0, \partial /$ $\partial y=0$ and $\partial / \partial x=0$, therefore, the conservation equations of gas phase can be described as follows:

$\partial\left(\alpha_{\mathrm{f}} \rho_{\mathrm{f}} w_{\mathrm{f}}\right) / \partial z=0$

$\partial\left(\alpha_{\mathrm{f}} \rho_{\mathrm{f}} w_{\mathrm{f}} u_{\mathrm{f}}\right) / \partial z=\partial \tau_{\mathrm{f}, z x} / \partial z-F_{D x}$, 


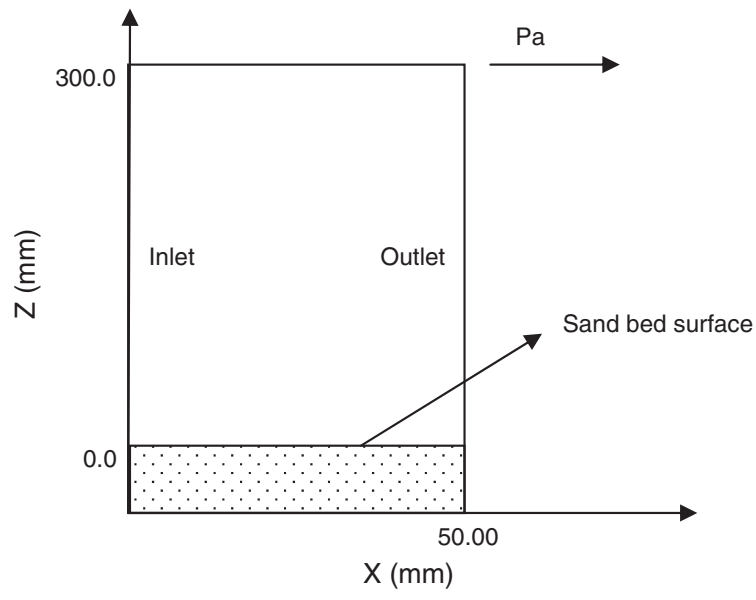

Fig. 1. Calculation domain.

where $\alpha_{\mathrm{f}}\left(=1-\alpha_{\mathrm{p}}\right), \rho_{\mathrm{f}}, \mathbf{v}_{\mathrm{f}}, \tau_{\mathrm{f}}$ and $\left(-F_{D x}\right)$ are gas volume fraction, gas density, gas velocity $\left(u_{\mathrm{f}}, v_{\mathrm{f}}\right.$ and $w_{\mathrm{f}}$ represent the velocity components in $x, y$, and $z$ directions respectively), stress tension and interaction forces between gas and particle (here, only drag force is considered), respectively. Because $w_{\mathrm{f}}=0$ when $z=0$, it can be concluded that $w_{\mathrm{f}}$ is equal to zero in the whole calculation domain according to Eq. (1). Thus, Eq. (2) can be simplified as

$\tau_{\mathrm{f}, z x}(z+\Delta z)-\tau_{\mathrm{f}, z x}(z) \approx F_{D x} \Delta z$

In particle-absent region, i.e. $\alpha_{\mathrm{f}}=1$, as a result $F_{D x}=0$. It can be obtained from Eq. (2) that $\tau_{\mathrm{f}, z x}(z+\Delta z)-\tau_{\mathrm{f}, z x}(z)=0$, which means that the gas shear stress is a constant in the whole particle-absent domain. Therefore, a constant shear stress $\tau_{0}$ is applied at the top of the calculation domain where the particle is almost absent. When sand particles start to move, $F_{D x} \neq 0$, and statistically $F_{D x}>0$ because the averaged velocity of particles is always less than the averaged gas velocity. By tracking every particle's movement, one can be found that its velocity will vary with time even though the statistically averaged velocity of particle phase has reached steady state. For the particle denoted as 's', its equation of motion can be expressed as

$$
\begin{aligned}
& m_{\mathrm{p}} \mathrm{d} \mathbf{v}_{s} / \mathrm{d} t=m_{\mathrm{p}} \mathbf{g}+\mathbf{f}_{D, s}+\sum_{r=1}^{N_{r}} \mathbf{f}_{s, r}, \quad s=1,2,3, \cdots \cdots, \\
& m_{\mathrm{p}} \mathrm{d} u_{s} / \mathrm{d} t=f_{D x, s}+\sum_{r=1}^{N_{r}} f_{x, s, r}, \quad s=1,2,3, \cdots \cdots
\end{aligned}
$$

where $m_{\mathrm{p}}, \mathbf{f}_{D, s}, \mathbf{f}_{s, r}, N_{r}$ and $\mathbf{g}$ are mass of particle, drag force, collision force (occurring when particle 's' collides with particle ' $r$ '), the total number of the particles colliding with particle ' $s$ ' and the acceleration of gravity (obviously, $g_{x}=0$ ), respectively. According to Newton's third law of motion, one can obtain

$F_{D x}=\left(\sum_{s=1}^{N_{i, j, k l}} f_{D x, s}\right) / V_{i, j, k}$

Since $F_{D x}>0$, as a whole, particles must be accelerated. However, when the aeolian sand movement reaches steady state, the averaged particle velocity also reaches steady state, but is not accelerated to catch gas velocity. This 'unreasonable' issue can be easily explained by introducing SPS. The difference of SPS applied at $z_{k}+\frac{1}{2} \Delta z$ and $z_{k}-\frac{1}{2} \Delta z$ eliminates the effect of $F_{D x}$, so the total force of particle group in $\Delta z$ is zero. Consequently, as a whole, particles are not accelerated.
Suppose that at $z=z_{k}$, there is a control volume with height of $\Delta z$ and bottom area of $A$. From $t=t_{l}$ to $t=t_{l}+\Delta t$, the momentum increment of the particle labelled ' $s$ ' is

$m_{\mathrm{p}}\left(u_{k, l+1, s}-u_{k, l, s}\right) / \Delta t=f_{D x, S}+\sum_{r=1}^{R_{k}} f_{X, S, r}+\sum_{r=1}^{R_{k+1}} f_{x, S, r}+\sum_{r=1}^{R_{k-\frac{1}{2}}} f_{X, S, r}$

where $N_{r}=R_{k}+R_{k+\frac{1}{2}}+R_{k-\frac{1}{2}}$, if particle ' $\mathrm{r}$ ' is also in the control volume, then it belongs to $R_{k}$; if particle ' $\mathrm{r}$ ' is at $z=z_{k+\frac{1}{2}}$, it belongs to $R_{k+\frac{1}{2}}$; if particle ' $r$ ' is at $z=z_{k-\frac{1}{2}}$, it belongs to $R_{k-\frac{1}{2}}$. The total momentum change of particles inside the volume is

$$
\begin{aligned}
& {\left[\sum_{s=1}^{N_{k, l}} m_{\mathrm{p}} u_{k, l+1, s}-\sum_{s=1}^{N_{k, l}} m_{\mathrm{p}} u_{k, l, s}\right]} \\
& =\left[\sum_{s=1}^{N_{k, l+1}} m_{\mathrm{p}} u_{k, l+1, s}-\sum_{s=1}^{N_{k, l}} m_{\mathrm{p}} u_{k, l, s}\right] \\
& \quad-\left[\sum_{s=1}^{N_{k, l}+1} m_{\mathrm{p}} u_{k, l+1, s}-\sum_{s=1}^{N_{k, l}} m_{\mathrm{p}} u_{k, l+1, s}\right]= \\
& =\Delta t V_{k} F_{D x}+\Delta t \sum_{s=1}^{N_{k, l}} \sum_{r=1}^{R+\frac{1}{2}} f_{x, s, r}+\Delta t \sum_{s=1}^{N_{k, l} \sum_{r=1}^{k-\frac{1}{2}} f_{X, s, r}}
\end{aligned}
$$

where $N_{k, l}$ and $N_{k, l+1}$ are the number of particles that captured in the control volume at $t=t_{l}$ and $t=t_{l+1}$, respectively. $N_{k, l}$ can be divided into three parts: (a) $N_{k, l}^{*}$, the number of particles that at $t=t_{l+1}$ still remain in the control volume; (b) $N_{k, l}^{-}, \downarrow$, the number of particles that at $t=t_{l+1}$ have already left the volume through the surface $z=z_{k-\frac{1}{2}}$; (c) $N_{k, l}^{+} \uparrow$, the number of particles that at $t=t_{l+1}$ have already left through the surface $z=z_{k+\frac{1}{2}}$. Thus,

$$
\begin{aligned}
N_{k, l}= & N_{k, l}^{*}+N_{k, l}^{-, \downarrow}+N_{k, l}^{+, \uparrow} \sum_{s=1}^{N_{k, l}} m_{\mathrm{p}} u_{k, l+1, s}=\sum_{s=1}^{N_{k, l}^{*}} m_{\mathrm{p}} u_{k, l+1, s} \\
& +\sum_{s=1}^{N_{k, l}^{-\downarrow}} m_{\mathrm{p}} u_{k, l+1, s}+\sum_{s=1}^{N_{k, l}^{+\uparrow}} m_{\mathrm{p}} u_{k, l+1, s}=M_{l, l+1}^{*} \\
& +M_{l, l+1}^{-, \downarrow}+M_{l, l+1}^{+, \uparrow}
\end{aligned}
$$

$N_{k, l+1}$ can also be divided into three parts: (a) $N_{k, l+1}^{*}$, the number of particles that at $t=t_{l}$ have already been in $V_{k}$; (b) $N_{k, i+1}^{-, \uparrow}$, the number of particles that at $t=t_{l}$ were out of the volume, but before $t=t_{l+1}$ went into the volume through the surface $z=z_{k-\frac{1}{2}}$; (c) $N_{k, i+1}^{+, \downarrow}$, the number of particles that at $t=t_{l}$ were out of the volume, but before $t=t_{l+1}$ went into the volume through the surface $z=z_{k+\frac{1}{2}}$. Note that $N_{k, l}^{*}$ is equal to $N_{k, l+1}^{*}$. Thus,

$$
\begin{aligned}
N_{k, l+1} & =N_{k, l+1}^{*}+N_{k, l+1}^{-, \uparrow}+N_{k, l+1}^{+, \downarrow} \sum_{s=1}^{N_{k, l+1}} m_{\mathrm{p}} u_{k, l+1, s} \\
& =\sum_{s=1}^{N_{k, l+1}^{*}} m_{\mathrm{p}} u_{k, l+1, s}+\sum_{s=1}^{N_{k, \uparrow+1}^{-\uparrow}} m_{\mathrm{p}} u_{k, l+1, s}+\sum_{s=1}^{N_{k, l+1}^{+, \downarrow}} m_{\mathrm{p}} u_{k, l+1, s} \\
& =M_{l, l+1}^{*}+M_{l, l+1}^{-, \uparrow}+M_{l, l+1}^{+, \downarrow}
\end{aligned}
$$

By introducing Eqs. (8) and (9) into Eq. (7), one can be obtained

$$
\begin{aligned}
& {\left[\sum_{s=1}^{N_{k, l+1}} m_{\mathrm{p}} u_{k, l+1, s}-\sum_{s=1}^{N_{k, l}} m_{\mathrm{p}} u_{k, l, s}\right]=\left(M_{l, l+1}^{+, \downarrow}-M_{l, l+1}^{+, \uparrow}\right)} \\
& \quad-\left(M_{l, l+1}^{-, \downarrow}-M_{l, l+1}^{-, \uparrow}\right)+\Delta t V_{k} F_{D x}+\Delta t \sum_{s=1}^{N_{k, l}} \sum_{r=1}^{k+\frac{1}{2}} f_{x, s, r} \\
& +\Delta t \sum_{s=1}^{N_{k, l}} \sum_{r=1}^{R}{ }_{r=1}^{k-1} f_{X, S, r}
\end{aligned}
$$


From $t=t_{l}$ to $t=t_{l+1}$, the net momentum flux through $z=z_{k+\frac{1}{2}}$ and $z=z_{k-\frac{1}{2}}$ are $\left(M_{l, l+1}^{+, \downarrow}-M_{l, l+1}^{+, \uparrow}\right)$ and $\left(M_{l, l+1}^{-,}-M_{l, l+1}^{-}, \uparrow_{1}\right)$, respectively. The momentum exchange due to particle collision at $z=z_{k+\frac{1}{2}}$ and $z=z_{k-\frac{1}{2}}$ are $\Delta t \sum_{s=1}^{N_{k, l}} \sum_{r=1}^{{ }^{k+1} \frac{1}{2}} f_{X, S, r}$ and $\Delta t \sum_{s=1}^{N_{k, l}} \sum_{r=1}^{{ }^{k}-\frac{1}{2}}\left(-f_{X, S, r}\right)$, respectively. If we summarize the momentum change into stresses, then

$\left(M_{l, l+1}^{+, \downarrow}-M_{l, l+1}^{+, \uparrow}\right)=A \Delta t \tau_{\mathrm{p}, z x}\left(z_{k+\frac{1}{2}}\right), \sum_{s=1}^{N_{k, l}} \sum_{r=1}^{k+\frac{1}{2}} f_{x, s, r}=A \tau_{\mathrm{col}, z x}\left(z_{k+\frac{1}{2}}\right)$

$\left(M_{l, l+1}^{-, \downarrow}-M_{l, l+1}^{-\uparrow \uparrow}\right)=A \Delta t \tau_{\mathrm{p}, z x}\left(z_{k-\frac{1}{2}}\right), \sum_{s=1}^{N_{k, l} \sum_{r=1}^{k-\frac{1}{2}}}\left(-f_{X, S, r}\right)=A \tau_{\mathrm{col}, z x}\left(z_{k-\frac{1}{2}}\right)$

where $\tau_{\mathrm{p}, z x}\left(z_{k+\frac{1}{2}}\right)$ and $\tau_{\mathrm{p}, z x}\left(z_{k-\frac{1}{2}}\right)$ are the SPS at $z=z_{k+\frac{1}{2}}$ and $z=z_{k-\frac{1}{2}}$, respectively; $\tau_{\text {col }, z x}\left(z_{k+\frac{1}{2}}\right)$ and $\tau_{\text {col }, z x}\left(z_{k-\frac{1}{2}}\right)$ are particle collision stress at $z=z_{k+\frac{1}{2}}$ and $z=z_{k-\frac{1}{2}}$, respectively.

The particle collision stress is negligible in calculation domain, except in the region very near to the sand bed, which is not discussed in this paper. Because of the steady state, $\left[\sum_{s=1}^{N_{k, l+1}} m_{\mathrm{p}} u_{k, l+1, s}-\right.$ $\left.\sum_{s=1}^{N_{k, l}} m_{\mathrm{p}} u_{k, l, s}\right]=0$. Neglecting particle collision stress and introducing Eq. (2) into Eq. (10), one can obtain

$\left[\tau_{\mathrm{f}, z x}+\tau_{\mathrm{p}, z x}\right]_{z=k+\frac{1}{2}}=\left[\tau_{\mathrm{f}, z x}+\tau_{\mathrm{p}, z x}\right]_{z=k-\frac{1}{2}}=\left[\tau_{\mathrm{f}, z x}+\tau_{\mathrm{p}, z x}\right]_{z=H}=\tau_{0}$

Eq. (13) indicates that in aeolian sand movement, gas shear stress varies along the vertical direction. However, the sum of the gas stress and the SPS remains unchanged along the height, and equals to the gas stress in particle-absent region. It should be pointed out that Eqs. (2)-(12) are reliable for all particle regions, but Eq. (13) is acceptable only under the condition that particle collision stress is negligible, i.e. in the dilute region where the particle volume fraction is less than $5 \%$ based on the calculations by this work. In the dense region, since the particle collision stress plays an important role, Eq. (13) should be reformed as follows

$$
\begin{aligned}
& {\left[\tau_{\mathrm{f}, z x}+\tau_{\mathrm{p}, z x}+\tau_{c}\right]_{z=k+\frac{1}{2}}=\left[\tau_{\mathrm{f}, z x}+\tau_{\mathrm{p}, z x}+\tau_{c}\right]_{z=k-\frac{1}{2}}} \\
& =\left[\tau_{\mathrm{f}, z x}+\tau_{\mathrm{p}, z x}+\tau_{c}\right]_{z=H}=\tau_{0}
\end{aligned}
$$

where $\tau_{c}$ is the particle collision stress. However, particle collision stress is beyond the scope of this paper.

\section{CFD-DEM simulations}

\subsection{Numerical method and simulating conditions}

A regular CFD-DEM (discrete element method) method was used to simulate aeolian sand movement, which is a typical E-L way to simulate dense two-phase flow. The calculation was unsteady. As the statistically averaged flow parameters reached steady state, the calculation was ceased.

The calculation domain is shown in Fig. 1. Initially, sand particles were uniformly distributed in the calculation domain and started to fall down to the bottom. After particle deposition a sand bed was automatically formed with a thickness. Particles impacting the sand bed were used to initiate the motion of sand particles on the surface of the sand bed. The bottom of the sand bed was treated as a fixed surface. All particles below that surface were assumed to be at rest at all times and were excluded in the calculation domain. The periodic boundary conditions were used for
Table 1

Cases.

\begin{tabular}{llc}
\hline No. & Particle diameter $(\mathrm{mm})$ & $\tau_{0}(\mathrm{~Pa})$ \\
\hline 1 & 0.33 & 14.7 \\
2 & 0.2 & 9.5 \\
3 & 0.2 & 7.5 \\
4 & 0.2 & 6.5 \\
\hline
\end{tabular}

the inlet and the outlet both in $\mathrm{X}$ and $\mathrm{Y}$ directions. When a particle leaves from the inlet or the outlet, it will enter through the other port. The simulation data in the steady state were carefully studied. The steady state means that the statistically averaged flow parameters do not change with time. In practical calculations the sand transport rate per unit width was monitored. When the aeolian sand movement reached the steady state, the sand transport rate per unit width did not change any longer.

In simulation, the gas density, dynamic viscosity of gas and particle density are $1.2 \mathrm{~kg} / \mathrm{m}^{3}, 1.785 \times 10^{-5} \mathrm{~Pa} \cdot \mathrm{s}$ and $2650 \mathrm{~kg} / \mathrm{m}^{3}$, respectively. The friction coefficient is 0.4 , the stiffness coefficient $1500 \mathrm{~N} / \mathrm{m}$, and the damping coefficient 0.002 . The restitution coefficient is 0.85 , which is determined by the damping and stiffness coefficients [7].

The computational time-steps were chosen as $2.0 \times 10^{-5} \mathrm{~s}$ for the fluid and $2.0 \times 10^{-6} \mathrm{~s}$ for particles. That is to say, there were 10 integration steps for the particle trajectory in every time-step for fluid motion [8]. Details of three dimensional CFD-DEM models can be found in reference [9].

In order to validate SPS and discuss its role in particle momentum transportation, four cases were selected to present in this paper, which are listed in Table 1.

\subsection{Results and discussion}

Fig. 2 gives the gas velocity distribution along height for case 1 . It can be seen that when there is no particle loading, gas velocity increases logarithmically with the height. When particle loading is introduced, gas velocity is greatly reduced. It implies that a part of gas momentum must be transferred into particle phase.

Fig. 3 shows that the gas shear stress and the total stress vary along height for different cases. It can be seen that for all the cases, with increment in height the gas shear stress increases, but the total stress almost keeps constantly and equals to $\tau_{0}$, except in the region very near to the sand bed (where particle collision stress cannot be neglected). These results validate the previous theoretical derivation that in aeolian sand movement gas stress is no more a constant, but the sum of gas shear stress and SPS are constant in the region almost without particle collision. This paper is focused on the derivation of

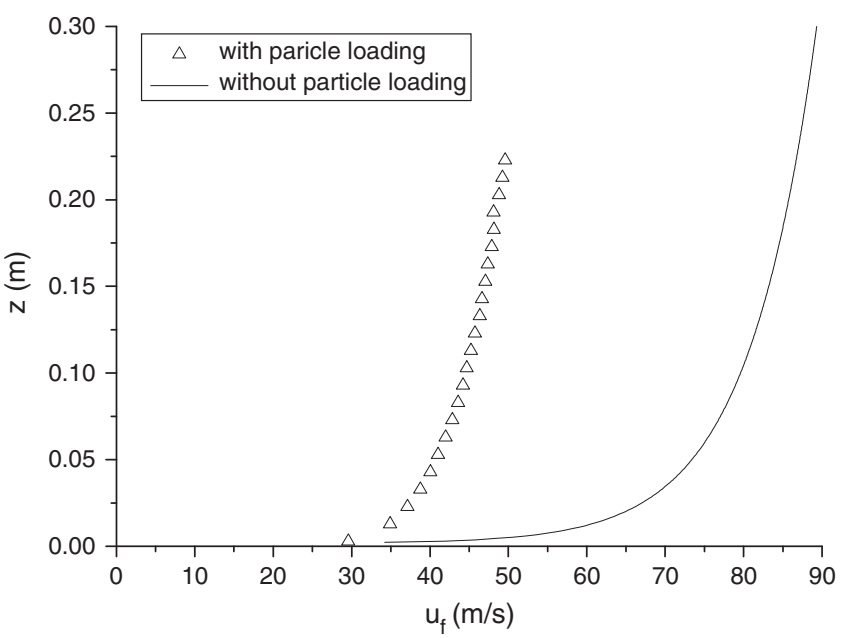

Fig. 2. Gas velocities of case 1. 
SPS. The details of CFD-DEM calculation and validation can be found in the author's previous published papers $[7,8]$.

Fig. 4 shows that SPS vary along height for different cases, which have the same particle diameter $(0.2 \mathrm{~mm})$ but are under different top gas shear stress (9.5 Pa, 7.5 Pa and 6.5 Pa). Based on the information in this figure, with an increasing height, the SPS sharply increases firstly and then gradually decreases. The higher top gas shear stress is, the higher SPS will be. The maximum SPS for case 2 and case 3 are $2.7 \mathrm{~Pa}$ and 2.0 Pa, respectively, while the maximum SPS for case 4 is $1.8 \mathrm{~Pa}$. Higher SPS implies that particle phase obtains more momentum from gas phase, thus particles will be transported at a longer distance. When flow reaches steady state, at a certain height, the number of particles moving downwards is statistically equal to that of particles moving upwards. However, in statistical significance, the particles moving downwards always carry more horizontal momentum than that particles moving upwards carry. Therefore, the direction of momentum transportation in particle phase should be downward.

\subsection{Conclusions}

1. The concept of SPS (statistical particle stress) is developed in this paper, which helps to understand the mechanism of momentum transportation in particle phase.

2. With increasing in height, gas shear stress increases while SPS decreases, but the sum of gas stress and SPS remains unchanged.

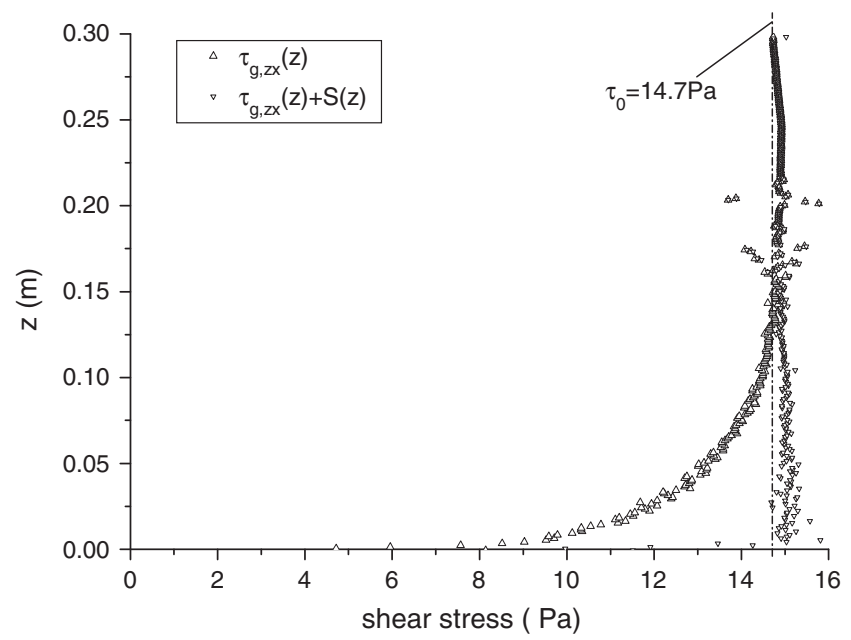

Case 1

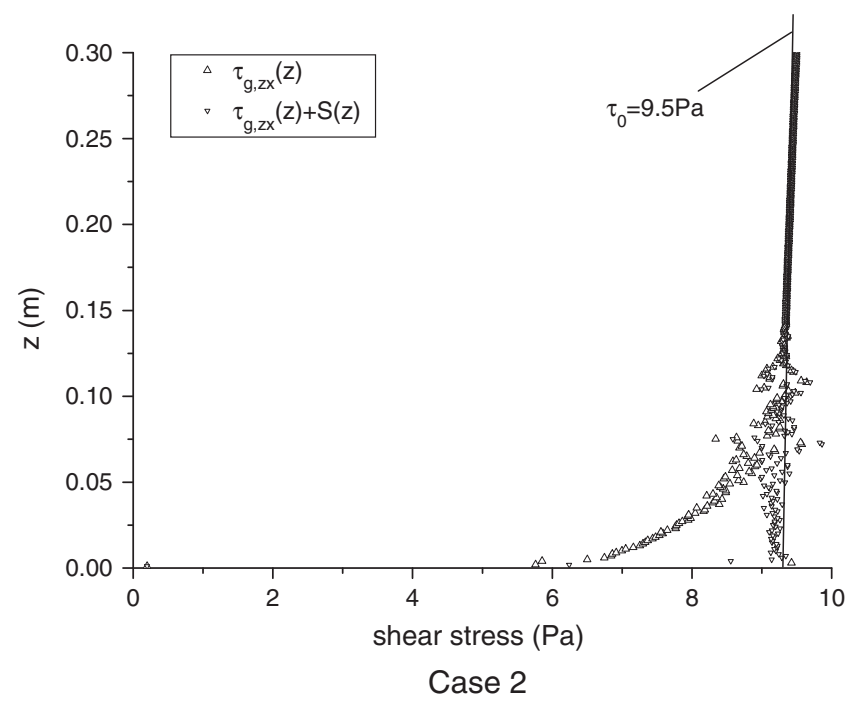

Fig. 3. Gas shear stress and total stress.

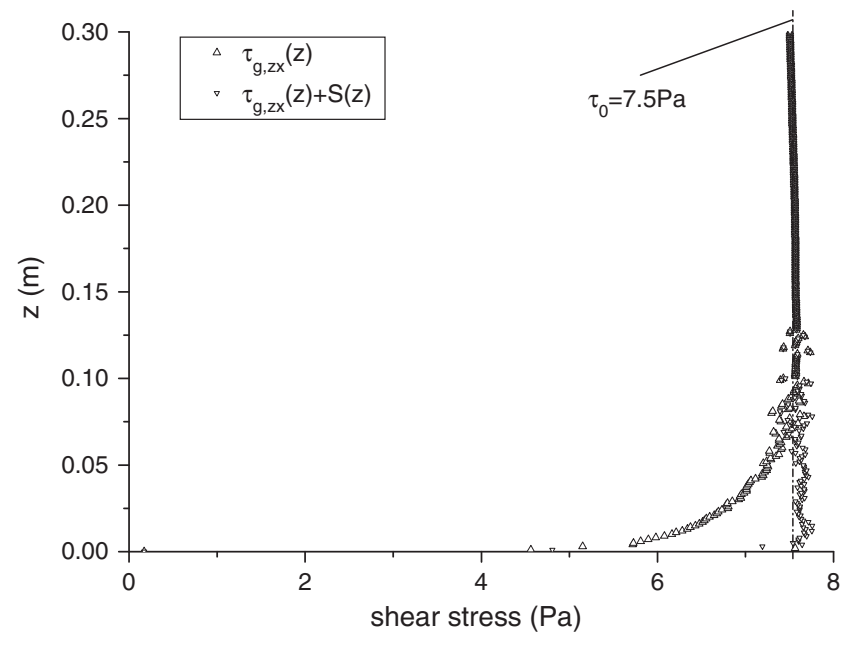

Case 3

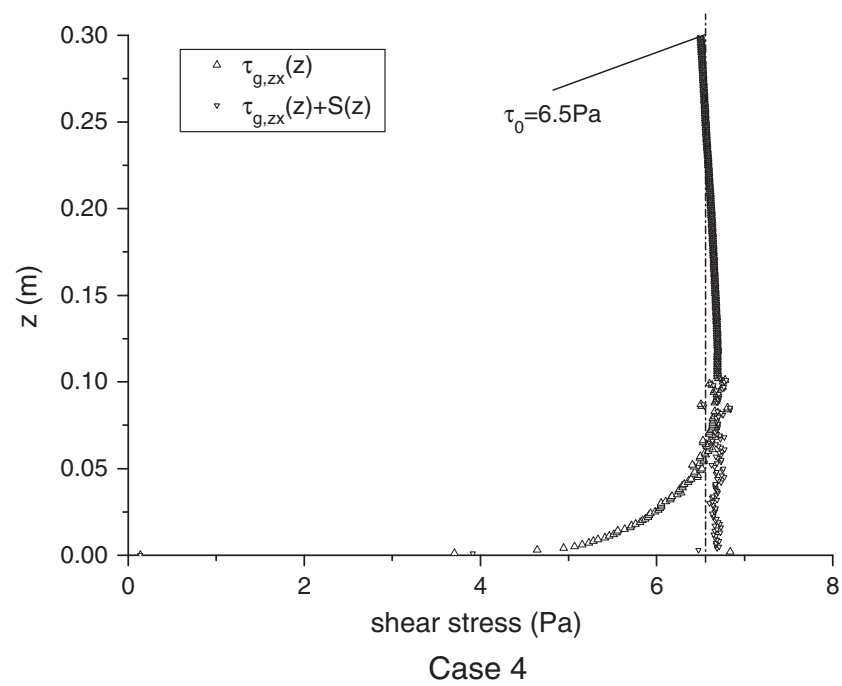

Fig. 3 (continued).

\section{Acknowledgements}

This research is sponsored by the National Natural Science Foundation of China (Grant No. 10972223) and the CAS Innovation Program.

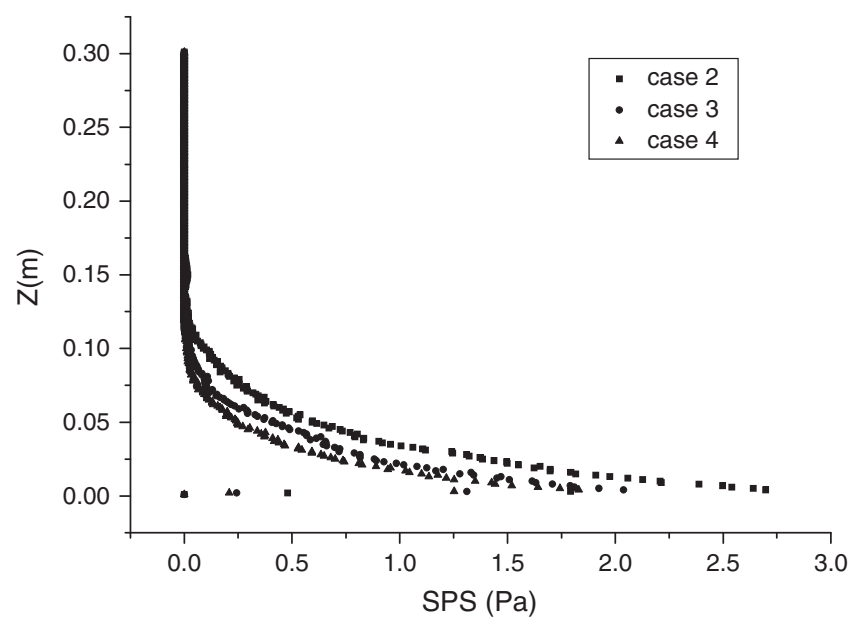

Fig. 4. SPS for different cases. 


\section{References}

[1] X.Y. Zou, Z.L. Wang, Q.Z. Hao, et al., The distribution of velocity and energy of saltating sand grains in a wind tunnel, Geomorphology 36 (2001) 155-165.

[2] Z.B. Dong, X.P. Liu, H.T. Wang, et al., The flux profile of a blowing sand cloud: a wind tunnel investigation, Geomorphology 49 (2002) 219-230.

[3] L.Q. Kang, L.J. Guo, D.Y. Liu, Experimental investigation of particle velocity distributions in windblown sand movement, Sci. China G Phys. Mech. Astron. 51 (2008) 986-1000.

[4] Qicheng Sun, Guangqian Wang, DEM application, to aeolian sediment transport and impact process in saltation, Part. Sci. Technol. 19 (2001) 339-353.
[5] Qicheng Sun, Guangqian Wang, Numerical simulation of aeolian sediment transport, Chin. Sci. Bull. 46 (9) (2001) 786-788.

[6] N. Huang, Y.L. Zhang, R. D'Adamo, A model of the trajectories and midair collision probabilities of sand particles in a steady state saltation cloud, J. Geophys. Res. 112 (2007) D08206.

[7] L. Kang, D. Liu, Numerical investigation of particle velocity distributions in aeolian sand transport, Geomorphology 115 (2010) 156-171.

[8] Jie-Cheng Yang, Yu Zhang, Da-You Liu, Xiao-lin Wei, CFD-DEM simulation of threedimensional aeolian sand movement, Sci. China (G) 53 (7) (2010) 1306-1318.

[9] EDEM Solution Manual. 